\title{
DIMENSIONAMENTO DE UM CIRCUITO DE CONDICIONAMENTO DE SINAIS PARA LEITURA COM MICROCONTROLADORES CONSIDERANDO A ELIMINAÇÃO DE RUÍDOS DE ALTA FREQUÊNCIA
}

\author{
DIMENSION OF A SIGNAL CONDITIONING CIRCUIT FOR READING WITH \\ MICROCONTROLLERS CONSIDERING THE ELIMINATION OF HIGH FREQUENCY NOISES
}

\section{DIMENSIÓN DE UN CIRCUITO DE ACONDICIONAMIENTO DE SEÑAL PARA LA LECTURA DE MICROCONTROLADOR CONSIDERANDO LA ELIMINACIÓN DE LOS RUIDO DE ALTA FRECUENCIA}

\author{
Leandro Paiter'; Cleidimar Nardi²; Felipe Marcon Toller ${ }^{3}$; Hiago Alves Teixeira ${ }^{4}$ \\ leandro.paiter@gmail.com \\ nardi.cleidimar@gmail.com \\ alemao_toller@hotmail.com \\ hiagoteixeira@live.com
}

\begin{abstract}
Resumo: Este trabalho apresenta o projeto de um circuito de condicionamento de sinais, onde o mesmo ajusta os sinais provenientes de sensores ou transdutores para os níveis de tensão aceitáveis em microcontroladores ou microprocessadores, suprindo ruídos de altas frequências. Para realizar deste processo é utilizado um circuito de instrumentação com ganho ajustável. Neste circuito de instrumentação também é possível inserir deslocamento ou offset positivo de tensão, também ajustável. Após o circuito de instrumentação, uma etapa de filtragem é inserida. Nesta etapa ocorre a atenuação de sinais em frequências indesejadas. A última etapa é uma circuito para casamento de impedâncias, que permite que o circuito de instrumentação não sofra interferências pelo dispositivo que irá ler os sinais condicionados.
\end{abstract}

Palavras-chave: Circuito condicionador sinais. Ajuste de ganho. Ajuste de deslocamento. Filtro ativo. Impedância.

\begin{abstract}
This work presents the design of a signal conditioning circuit, where it adjusts signals from sensors or transducers to acceptable voltage levels in microcontrollers or microprocessors, supplying high frequency noise. To perform this process an adjustable gain instrumentation circuit is used. In this instrumentation circuit it is also possible to insert positive voltage offset or offset, also adjustable. After the instrumentation circuit, a filtering step is inserted. At this stage attenuation of signals occurs at unwanted frequencies. The last step is an impedance matching circuit, which allows the instrumentation circuit to not be disturbed by the device that will read the conditioned signals.
\end{abstract}

Key words: Circuit conditioner signs. Gain adjustment. Offset adjustment. Filter active. Impedance.

Resumen: Este trabajo presenta el diseño de un circuito de acondicionamiento de señales, donde ajusta las señales de los sensores o transductores a niveles de voltaje aceptables en microcontroladores o microprocesadores, suministrando ruido de alta frecuencia. Para realizar este proceso se utiliza un circuito de instrumentación de ganancia ajustable. En este circuito de instrumentación también es posible insertar una compensación de voltaje positivo o una compensación, también ajustable. Después del circuito de instrumentación, se inserta un paso de filtrado. En esta etapa, la atenuación de las señales se produce en frecuencias no deseadas. El último paso es un circuito de adaptación de impedancia, que permite que el circuito de instrumentación no sea perturbado por el dispositivo que leerá las señales condicionadas.

Palabras llave: Señales de acondicionamiento de circuito. Ajuste de ganancia. Ajuste de compensación. Filtro activo. Impedancia 


\section{INTRODUÇÃO}

As atividades de monitoramento de grandezas físicas, automação de processos industriais e controle de sistemas eletrônicos são dependentes de aquisição de dados para auxilio nas tomadas de decisões, sejam elas computacionais ou não. Em (DELLA FLORA, 2009), (NARDI, 2016) e (PAITER, 2015), são apresentados exemplos de aplicações em que a aquisição de dados se faz necessário.

Nas aplicações apresentadas em (DELLA FLORA, 2009), (NARDI, 2016) e (PAITER, 2015) são utilizados circuitos de condicionamento de sinais para adequar o sinal lido por meio de sensores ou transdutores às necessidades dos dispositivos que irão interpretar e tomar decisões com base nestas informações. Nestes três casos os circuitos de instrumentação foram projetados com características exclusivas do projeto ao qual estavam inseridos.

Muitas outras aplicações necessitam de medições realizadas por meio de sensores ou transdutores. Em alguns casos os sensores já estão encapsulados com circuitos que realizam um pré-condicionamento de sinais, como é o caso do sensor de temperatura LM35. Este sensor é largamente utilizado para fins didáticos e em sistemas que se deseja verificar a temperatura de um sistema sem maiores dificuldades, uma vez que o pré-condicionamento garante uma tensão de saída linear em toda a escala de $10 \mathrm{mV} /{ }^{\circ} \mathrm{C}$ (NATIONAL SEMICONDUCTOR, 2000).

A saída desse sensor poderia ser facilmente convertida por um conversor analógico/digital e utilizada em um microcontrolador para tomada de decisões. Entretanto, outros sensores não possuem tais circuitos de pré-condicionamento, como é o caso do thermistor NTC, que somente varia sua resistência com a alteração de temperatura (MURATA MANUFACTURING CO., LTD., 2013), necessitando de um circuito auxiliar para realizar a conversão da variação de temperatura em variação de tensão e então ser lido por um conversor $A D$.

Em casos em que se necessita realizar o condicionamento de sinais ou amplificação de sinais é necessário um circuito que seja capaz de realizar esta função. Este artigo apresenta as etapas de dimensionamento e execução de um circuito de instrumentação e condicionamento de sinais para as mais diversas aplicações. Este circuito, projetado e construído de forma didática, realiza a amplificação e o condicionamento em etapas bem definidas para que seja possível realizar as alterações necessárias em função de cada aplicação.

A primeira etapa é o circuito de ganho de tensão, em que ocorre a amplificação da diferença de potencial aplicado às entradas do circuito. A segunda etapa é o deslocamento de tensão, para que possa ser possível operar com sinais de entrada alternados e garantir saída somente positiva. A terceira etapa é a filtragem, que atenua sinais de frequências indesejadas. A última etapa é o casamento de impedâncias, necessário para garantir que o circuito de instrumentação não sofra interferência dos circuito que estará conectado a sua saída. Cada uma destas etapas será detalhada no decorrer do artigo. 


\section{prociênci@s}

\section{v. 2, n. 2, dezembro, 2019}

\section{O CIRCUITO DE CONDICIONAMENTO DE SINAIS}

Nesta seção descrevem-se os métodos adotados para o dimensionamento do circuito de condicionamento de sinais, além da descrição dos materiais utilizados para a elaboração do circuito utilizado para realização dos ensaios experimentais.

\subsection{Amplificador de instrumentação}

O amplificador de instrumentação é um circuito que possui como uma das principais características o elevado ganho de tensão diferencial das portas de entrada, ou seja, possui a capacidade de amplificar significativamente a diferença de potência aplicada em suas portas de entrada (MALVINO e BATES, 2016). Outras características importantes deste circuito são a elevada resistência de entrada e baixa resistência de saída, elevada razão de rejeição de modo comum, elevado ganho de tensão em malha aberta, baixa tensão de offset de entrada e drift extremamente baixo, o que é uma característica importante em casos onde ocorre elevadas variações de temperatura (PERTENCE JUNIOR, 2015).

Este circuito será responsável pelo ajuste do ganho e por meio dele será inserido o deslocamento positivo da tensão, ou tensão de offset como conhecido na literatura. A forma construtiva de um circuito de instrumentação é representado na Figura 1.

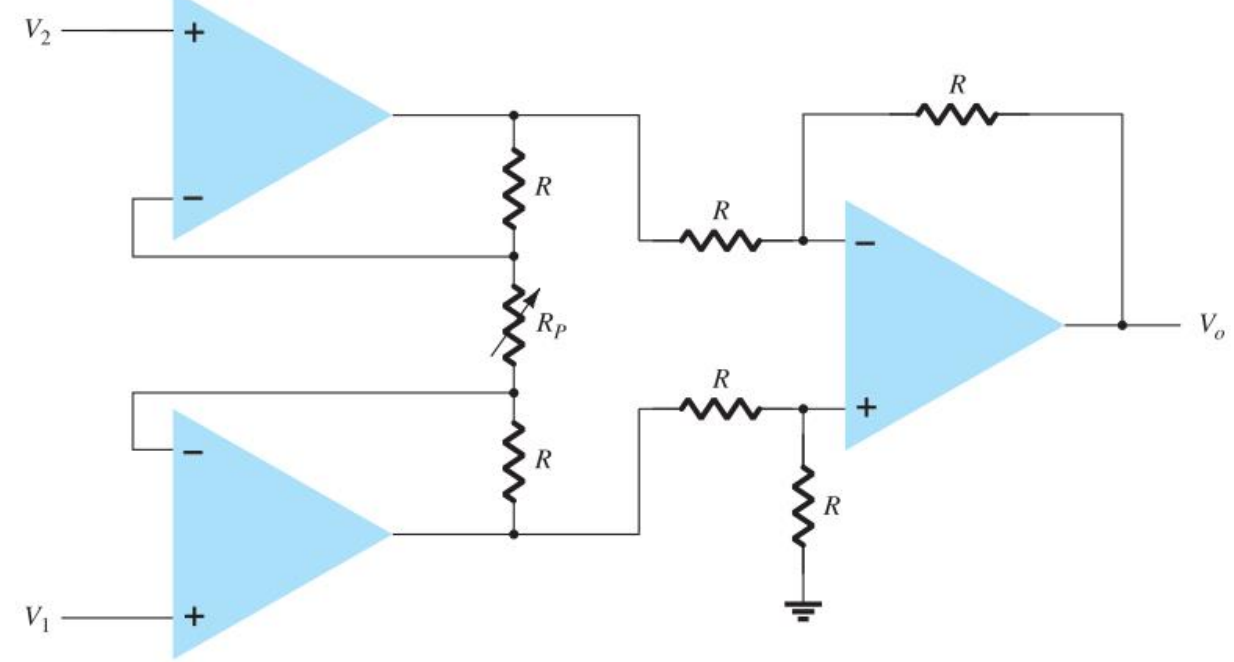

Figura 1. Representação da estrutura de um circuito amplificador de instrumentação.

Fonte: (BOYLESTAD e NASHELSKY, 2013).

O valor da tensão de saída em função da diferença de potencial aplicado às entradas é descrito por (1).

$$
V_{o}=\left(V_{1}-V_{2}\right)\left(1+\frac{2 R}{R_{p}}\right)
$$

Outra vantagem deste circuito é a facilidade de alteração do ganho de tensão, uma vez que ente ganho pode ser ajustado somente com a alteração do valor de $R_{p}$, como mostrado em (1). A elevação do valor de $R_{p}$ implicará na diminuição do ganho de tensão e a diminuição de $R_{p}$ irá elevar o ganho. Assim, é possível ajustar o ganho conforme as necessidades do projeto utilizando um resistor variável ou potenciômetro. 


\subsection{Deslocamento de tensão}

O amplificador de instrumentação possui outra vantagem significativa na possibilidade de inserir um deslocamento constante na tensão de saída. Este deslocamento pode ser positivo ou negativo e é inserido por meio de uma fonte de tensão constante conectada no ponto de aterramento do circuito representado na Figura 1. Desta forma, pode-se incluir o efeito do deslocamento reescrevendo (1) chegando ao resultado expresso em (2).

$$
V_{o}=\left(V_{1}-V_{2}\right)\left(1+\frac{2 R}{R_{p}}\right)+V_{o f f s e t}
$$

\subsection{Filtro ativo}

Um filtro elétrico é um quadripolo capaz de atenuar determinadas frequências do espectro do sinal de entrada e permitir a passagem das demais (PERTENCE JUNIOR, 2015). Um filtro elétrico pode ser construído com resistores, capacitores e indutores (BOYLESTAD e NASHELSKY, 2013), (MALVINO e BATES, 2016) e (ALEXANDER e SADIKU, 2013). Para melhorar os resultados obtidos e as características do filtro, pode-se incluir um amplificador operacional como dispositivo ativo e assim construir um filtro ativo (MANCINI, 2002). A utilização de filtros ativos apresenta vantagens como a eliminação de indutores e, facilidade e flexibilidade na implementação de projetos, mesmo em projetos mais complexos, uma vez que permite associação em cascata de vários estágios (PERTENCE JUNIOR, 2015).

Para realizar o dimensionamento de um filtro ativo torna-se necessário conhecer as características do filtro, como função executada, a topologia do circuito e função resposta (PERTENCE JUNIOR, 2015). Algumas literaturas como (THEDE, 2004), (PAARMANN, 2003) e (WINDER, 2002) apresentam técnicas de dimensionamento de filtros para as mais variadas aplicações. Neste trabalho será utilizado o método descrito em (PERTENCE JUNIOR, 2015). Neste artigo utiliza-se a topologia de segunda ordem fonte de tensão controlada por tensão ou Sallen-Key. Optou-se por esta topologia pela facilidade de utilização e pela não inversão da fase do sinal de saída na banda de passagem.

A estrutura de implementação de um filtro ativo passa baixas de 2aㅡ ordem com estrutura Sallen-Key é mostrada na Figura 2.

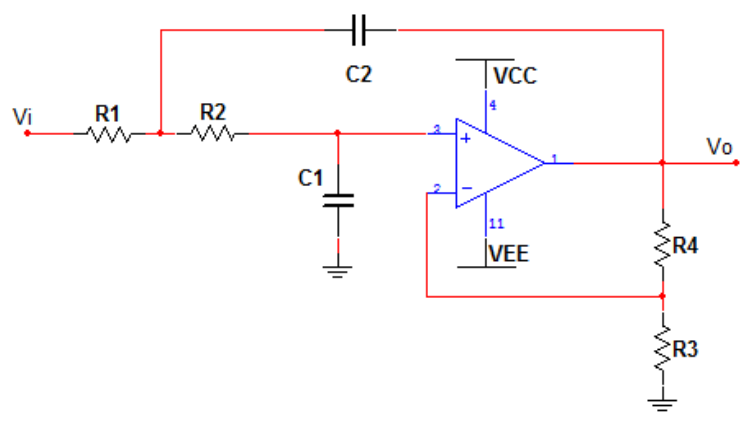

Figura 2. Representação de filtro ativo VCVS de segunda ordem.

Fonte: (BOYLESTAD e NASHELSKY, 2013). 


\section{prociênci@s}

\section{v. 2, n. 2, dezembro, 2019}

Para realização do dimensionamento desta topologia de filtro, necessita-se definir o ganho de tensão para o filtro, a frequência de corte $f_{c}$ e a aproximação ou função-resposta desejada. Com isto e seguindo o método apresentado em (PERTENCE JUNIOR, 2015), as equações para o dimensionamento são descritas em (3) e (4).

$$
\begin{gathered}
C_{2}=\frac{10}{f_{c}}[\mu F] \\
C_{1} \leq \frac{\left(a^{2}+4 b(K-1)\right) C_{2}}{4 b}
\end{gathered}
$$

Deve-se observar a desigualdade de (4). Caso a desigualdade não seja respeitada, as equação de dimensionamento apresentarão valores complexos.

Para a determinação dos valores das resistências, são utilizados as expressões descritas em (5), (6), (7) e (8).

$$
\begin{gathered}
R_{1}=\frac{2}{\left(a C_{2}+\sqrt{\left(a^{2}+4 b(K-1)\right) C_{2}^{2}-4 b C_{1} C_{2}}\right) \omega_{c}} \\
R_{2}=\frac{1}{b C_{1} C_{2} R_{1} \omega_{c}^{2}} \\
R_{3}=\frac{K\left(R_{1}+R_{2}\right)}{K-1} . \\
R_{4}=K\left(R_{1}+R_{2}\right) .
\end{gathered}
$$

Os valores de $a$ e $b$ em (4), (5) e (6) são determinados a partir da tabela para a aproximação desejada.

Em (5) e (6), utiliza-se

$$
\omega_{c}=2 \pi f_{c}
$$

Para os casos de ganho de tensão unitário, ou seja, $K=1$, R3 deverá ser um circuito aberto e R4 um curto-circuito. 


\section{prociênci@s}

\section{v. 2, n. 2, dezembro, 2019}

\subsection{Casamento de impedâncias}

O casamento de impedância pode ser realizado com um circuito que possui elevada impedância de entrada e baixa impedância de saída. Isto é conseguido com um circuito buffer de tensão que fornece um meio de isolar um sinal de entrada de uma carga usando um estágio com ganho de tensão unitário e sem inversão de fase ou polaridade (BOYLESTAD e NASHELSKY, 2013), (MALVINO e BATES, 2016) e (PERTENCE JUNIOR, 2015). A ligação do amplificador operacional para operar como buffer de tensão é representada na figura 3. Neste caso, o sinal de saída é igual ao sinal de entrada.

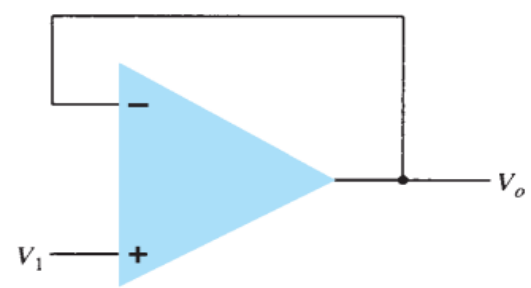

Figura 3. Representação de um amplificador operacional para casamento de impedâncias.

Fonte: (BOYLESTAD e NASHELSKY, 2013).

\section{CIRCUITO PRÁTICO DE CONDICIONAMENTO DE SINAIS}

O circuito prático para condicionamento de sinais é representado na Figura 4. Nesta representação estão o amplificador de instrumentação, circuito de deslocamento positivo de tensão, filtro ativo Sallen-Key e circuito de casamento de impedâncias.

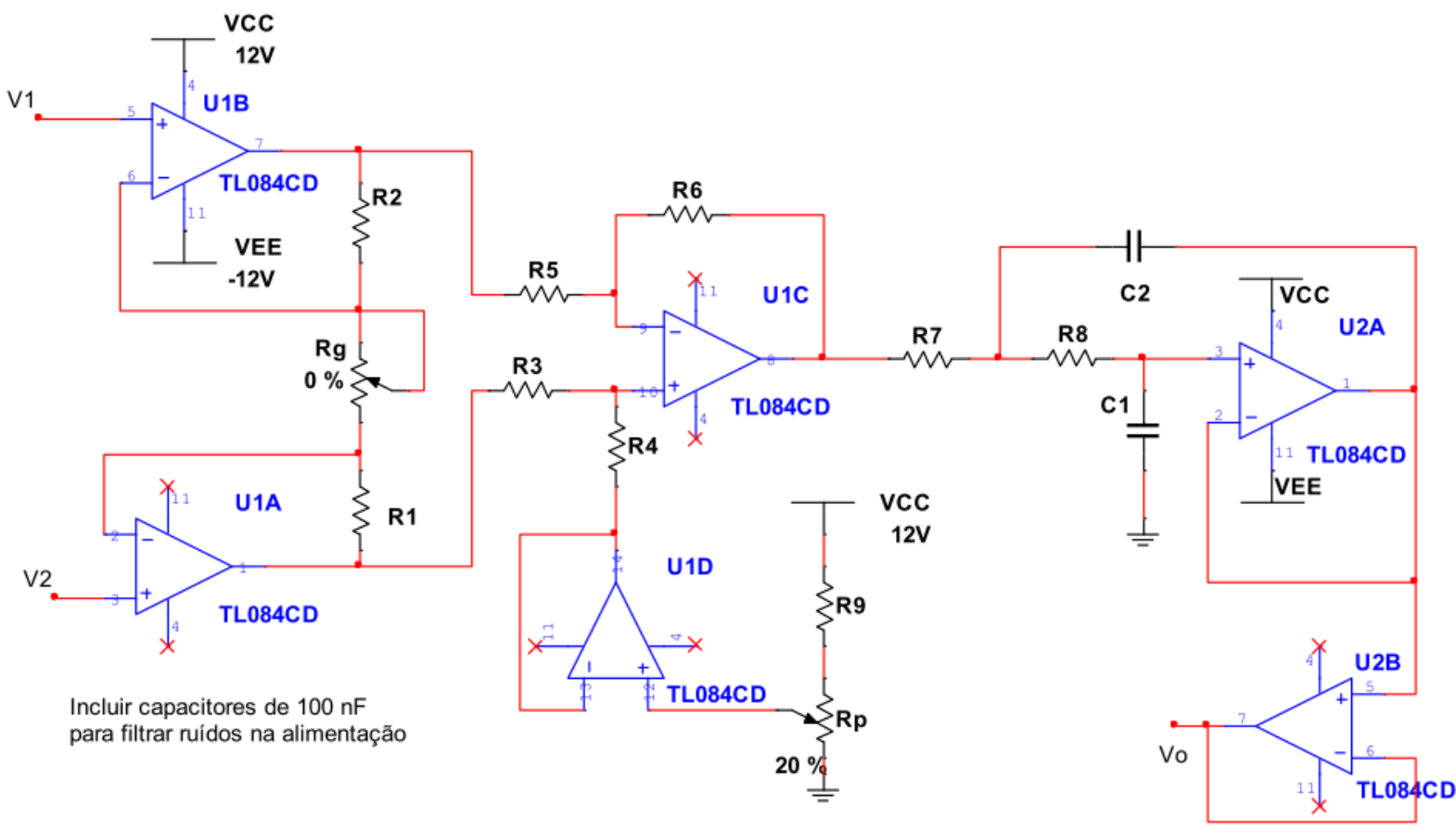

Figura 4-Ilustração do Esquema do Circuito de condicionamento de sinais.

Fonte: Autoria Própria 
As especificações de projeto para este circuito alimentado em 12 volts são:

- Máxima diferença de potencial de entrada $\pm 2 \mathrm{~V}$;

- Tensão máxima de saída 3,3 V ou $5 \mathrm{~V}$;

- Tensão mínima de saída $0 \mathrm{~V}$;

- Frequência de corte de $1200 \mathrm{~Hz}$.

Estes parâmetros são utilizados para dimensionar o circuitos. Para o circuito amplificador de instrumentação utilizou-se resistores de $10 \mathrm{k} \Omega$ em R1, R2, R3, R4, R5 e R6. Com os valores definidos para as resistências, o valor da resistência máxima para o potenciômetro é definida para o pior cenário. Neste caso, o menor ganho implicará na maior resistência. Aplicando os valores de $V_{0}=3,3 \mathrm{~V}, \mathrm{~V}_{1}-\mathrm{V}_{2}=2 \mathrm{~V}$ e $\mathrm{R}=10 \mathrm{k} \Omega$ em (1), obtém-se um valor de $30,7 \mathrm{k} \Omega$ para a resistência do potenciômetro $R_{G}$. Um valor comercial encontrado facilmente é o de $50 \mathrm{k} \Omega$ e este foi escolhido para compor o projeto. Assim, caso seja necessário alterar o ganho, basta alterar a posição da excursão do potenciômetro.

Para o circuito de deslocamento de tensão são escolhidos resistor de $15 \mathrm{k} \Omega$ para R9 e potenciômetro de $10 \mathrm{k} \Omega$ para Rp. Assim, a tensão máxima inserida para deslocamento está próxima a $5 \mathrm{~V}$ que é o limite máximo para a tensão de saída.

Para o filtro são escolhidos ganho unitário, uma vez que todo o ganho será inserido pelo circuito amplificador de instrumentação. Optou-se por uma resposta plana para o filtro passa baixas e uma das funções resposta mais utilizadas é a Butterworth. Consultando a tabelas para esta função respostas, os valores para as constantes a e b são $a=\sqrt{2}$ e $b=1$. Com a frequência de corte já definida, obtém-se os valores mostrados na Tabela 1.

Tabela 1. Valores comerciais dos componentes utilizados para construção do circuito de instrumentação.

\begin{tabular}{|c|c|c|c|c|c|c|c|c|c|c|c|c|c|}
\hline Componente & $\mathrm{R}_{\mathrm{G}}$ & $\mathrm{R}_{\mathrm{p}}$ & $\mathrm{R} 1$ & $\mathrm{R} 2$ & R3 & R4 & R5 & R6 & R7 & $\mathrm{R} 8$ & R9 & $\mathrm{C} 1$ & $\mathrm{C} 2$ \\
\hline \multirow[t]{2}{*}{ Valor } & 50 & 10 & 10 & 10 & 10 & 10 & 10 & 10 & 15 & 27 & 15 & 10 & 4,7 \\
\hline & $\mathrm{k} \Omega$ & $\mathrm{k} \Omega$ & $\mathrm{k} \Omega$ & $\mathrm{k} \Omega$ & $\mathrm{k} \Omega$ & $\mathrm{k} \Omega$ & $\mathrm{k} \Omega$ & $\mathrm{k} \Omega$ & $\mathrm{k} \Omega$ & $\mathrm{k} \Omega$ & $\mathrm{k} \Omega$ & $\mathrm{nF}$ & $\mathrm{nF}$ \\
\hline
\end{tabular}

Utilizou-se amplificadores operacionais modelo TL084 encapsulados em circuito integrado para construção dos circuitos e obtenção dos resultados. Próximo aos pinos de alimentação do circuito integrado incluiu-se capacitores cerâmicos de $100 \mathrm{nF}$ para filtragem de possíveis ruídos contidos na tensão CC de alimentação.

Para realizar a montagem da placa de circuito impresso foi utilizado o software Proteus para a criação do esquemático e layout, conforme ilustrado na Figura 5. 


\section{prociênci@s v. 2, n. 2, dezembro, 2019}

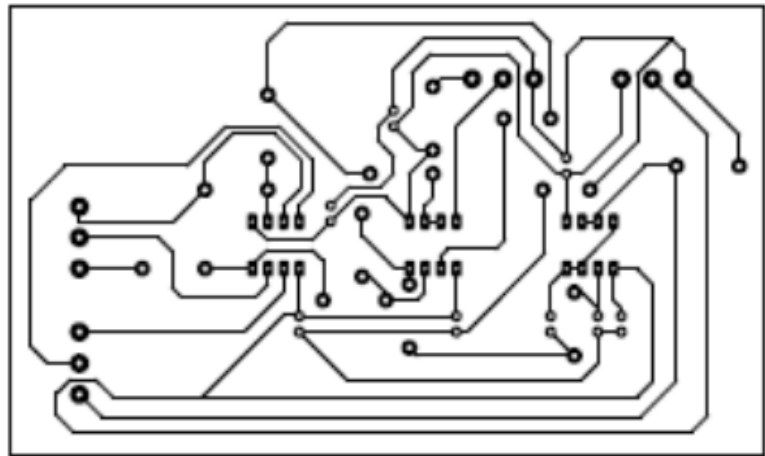

Figura 5 - Ilustração do esquema das Trilhas para a placa de circuito impresso.

Fonte: Autoria Própria

Posteriormente, utilizando técnica artesanal por meio térmico com auxílio de um ferro de passar e papel fotográfico, realizou-se a transferência deste circuito desenhado para uma placa de fenolite cobreada face simples. Após realizar a corrosão com percloreto de ferro e perfuração, soldou-se os componentes à placa e então realizou-se os testes. A placa finalizada é ilustrada por meio da Figura 6.

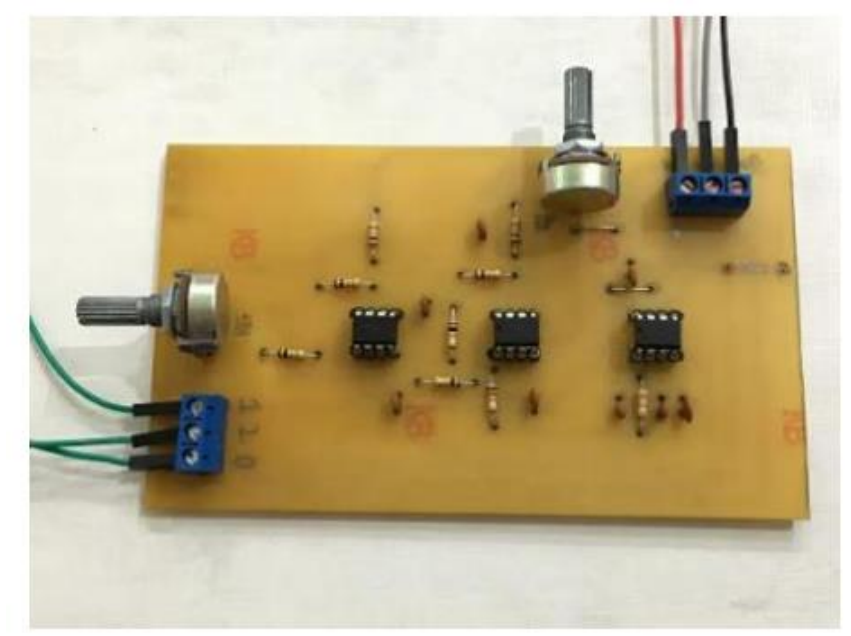

Figura 6 - Ilustração da placa de circuito impresso finalizada.

Fonte: Autoria Própria

Após o desenvolvimento do circuito apresentado na Figura 6, foram realizados ensaios com o objetivo de validar a proposta.

\section{RESULTADOS E DISCUSSÕES}

Para constatar o funcionamento do circuito, inicialmente verificou-se as possibilidades de variação de ganho. Este procedimento foi realizado aterrando o ponto V1 do circuito da Figura 4 e inserindo-se em V2, sinais senoidais alternados com frequência de $100 \mathrm{~Hz}$, partindo de $100 \mathrm{mV}$ e incrementado de $100 \mathrm{mV}$ até alcançar a diferença máxima de entrada de 2 V. Para cada valor de entrada, ajustava-se o potenciômetro para conseguir 
valores de 3,3 V e $5 \mathrm{~V}$ na saída. Com isto verificou-se na prática que é possível realizar ajuste de ganho para diversas combinações de tensão de entrada e saída.

Após isto, testou-se o circuito de deslocamento de tensão. Este teste foi realizado sem tensão de entrada. Assim, todo o circuito de deslocamento tornou-se responsável por todo o sinal de saída. Este circuito comportou-se como esperado variando a tensão de saída de zero a um valor um pouco acima de 4,8 V, não chegando aos 5 volts, uma vez que, devido aos valores escolhidos para $\mathrm{R} 9$ e $\mathrm{R}_{\mathrm{P}}$ isto não deveria ocorrer. Na Figura 7 é representado um resultado para um circuito com ganho de aproximadamente duas vezes e deslocamento de aproximadamente $2,4 \mathrm{~V}$.

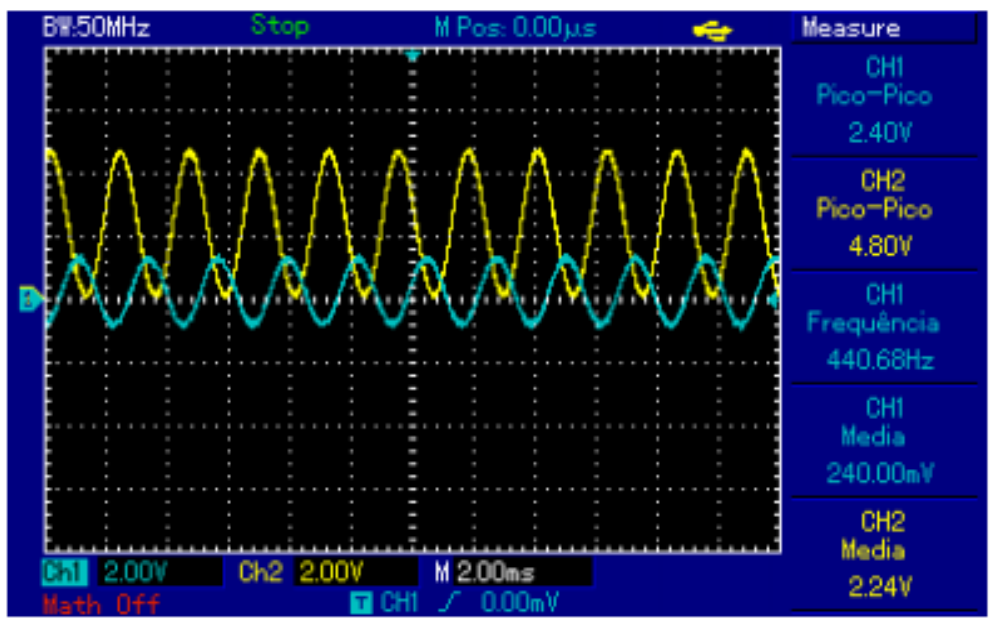

Figura 7. Resultado experimental para ganho e deslocamento de tensão verificando o sinal de entrada em $\mathrm{CH} 1$ e saída em $\mathrm{CH} 2$.

Fonte: Autoria Própria

Para analisar experimentalmente a resposta do circuito inseriu-se um sinal senoidal de $2 \mathrm{~V}_{\mathrm{PP}}$ e amplificou-se este sinal para atingir aproximadamente 3,3 V. Então realizou-se a leitura deste sinal na entrada do filtro com o canal $1(\mathrm{CH} 1)$ do osciloscópio configurado em acoplamento $A C$ e na saída do filtro como o canal $2(\mathrm{CH} 2)$ do osciloscópio configurado no acoplamento DC. Os resultados estão representados na Figura 8. 


\section{prociênci@s v. 2, n. 2, dezembro, 2019}

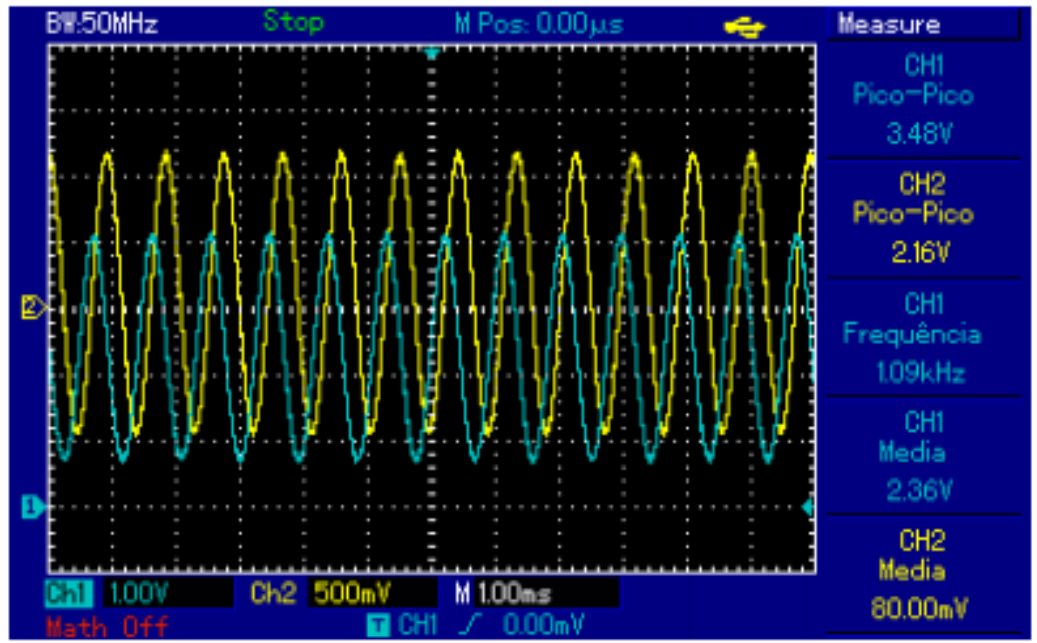

Figura 8 - llustração do resultado para frequência de corte com sinal de entrada em $\mathrm{CH} 1$ e sinal de saída em $\mathrm{CH} 2$, na frequência de $1090 \mathrm{~Hz}$.

Fonte: Autoria Própria

Com os resultados obtidos verificou-se que a frequência de corte ficou abaixo do valor desejado. Em (PERTENCE JUNIOR, 2015) estabelece-se que na frequência de corte o valor da tensão de saída pico a pico será menor em uma razão de $\sqrt{2}$ em relação a entrada. Desta forma, na frequência de $1090 \mathrm{~Hz}$ a tensão de saída já está abaixo do valor esperado. Esta diferença pode ser ocasionada pela não utilização de componentes com os valores equivalentes aos calculados, uma vez que foram utilizados valores comerciais disponíveis no laboratório. Outra justificativa para esta discrepância se dá pela utilização do osciloscópio que não é um equipamento confiável para obtenção dos valores. Os mesmos comparativos foram realizados utilizando valores adquiridos com multímetro mostraram maior proximidade entre o a frequência de corte de projeto e frequência de corte obtida.

Outro teste realizado ocorreu com inserção de sinal na porta V1 do circuito da Figura 4. Com as práticas realizadas verificou-se que para o sinal inserido nesta porta o circuito comporta-se de maneira similar aos resultados já descritos. A única diferença considerável é a inversão de fase, já esperada pelo comportamento descrito em (1). $\mathrm{Na}$ Figura 9, temos a demonstração do comportamento inserindo um sinal com frequência de $60 \mathrm{~Hz}$, tendo amplificação e deslocamento. Nesta figura fica a oposição de fase do sinal de saída mostrado em $\mathrm{CH} 2$ e entrada mostrado em $\mathrm{CH} 1$. 


\section{prociênci@s v. 2, n. 2, dezembro, 2019}

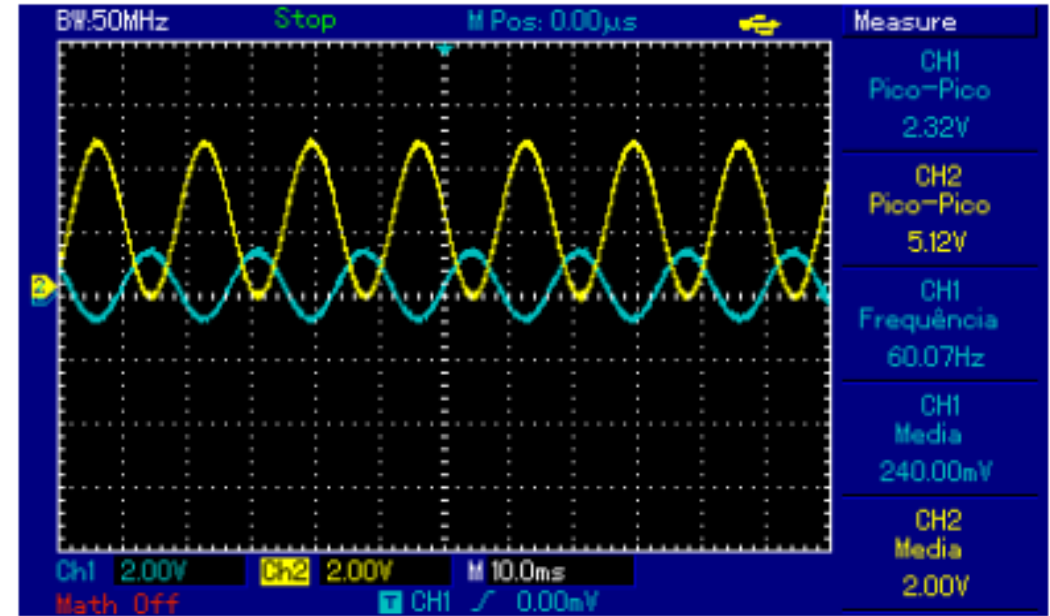

Figura 9 - Ilustração do resultado experimental para tensão de entrada (CH1) aplicado ao lado inversor do amplificador de instrumentação e saída $(\mathrm{CH} 2)$ do circuito de condicionamento de sinais.

Fonte: Autoria Própria

Desta forma foi possível validar de forma experimental a aplicação dos circuito para o fim desejado.

\section{CONCLUSÕES}

Os resultados obtidos demonstraram que as etapas de amplificação de sinal operou de acordo com o esperado, uma vez que foi possível alterar o ganho para diferentes valores de entrada de sinal, tanto aplicados a porta inversora como aplicados a porta não inversora do amplificador de instrumentação.

O circuito de deslocamento de tensão também realizou sua função conforme projetado, já que inseriu níveis de tensão para a saída do circuito sem exceder a tensão máxima de saída definida em projeto.

O circuito de casamento de impedância possui operação simples e não necessita de testes complexos. O único teste realizado comparou os sinais de entrada e saída deste circuito e, como se mostraram idênticos, confirmou a operação do circuito.

Apesar de bons resultados nestas três etapas, a etapa de filtro não apresentou resultado satisfatório, uma vez que sua frequência de corte se situou quase $200 \mathrm{~Hz}$ abaixo do valor estipulado. Esta diferença é significativa e em algumas aplicações não são toleráveis. Entretanto, como a metodologia utilizada para o dimensionamento é consolidada, justificase estas discrepância em função da utilização de componentes com valores significativamente diferentes dos calculados.

Apesar do circuito de filtragem não comportar-se como o esperado, julga-se o trabalho como atendendo aos parâmetros estabelecidos, já que didaticamente engloba diversos conceitos. Melhorias podem ser implementadas para dar maior flexibilidade ao projeto. Dentre elas, pode-se citar a implantação de um diodo zener e um retificador de precisão para garantir que a tensão de saída sempre esteja dentro dos limites estabelecidos, mesmo quando ocorram erros de ganho, deslocamento ou tensão de entrada. 


\section{prociênci@s}

\section{v. 2, n. 2, dezembro, 2019}

\section{REFERÊNCIAS}

ALEXANDER, C. K.; SADIKU, M. N. O. Fundamentos de Circuitos Elétricos. 5a . ed. Porto Alegre: AMGH, 2013. $874 \mathrm{p}$.

BOYLESTAD, R. L.; NASHELSKY, L. Dispositivos Eletrônicos e Teoria de Circuitos. 11a․ ed. São Paulo: Pearson Education, 2013.

DELLA FLORA, L. Contribuição ao controle de máquinas de vibração eletrodinâmicas. Santa Maria: [s.n.], 2009. 280 p.

MALVINO, A. P.; BATES, D. Eletrônica. 8ª . ed. Porto Alegre: AMGH, v. 1, 2016.

MANCINI, R. Op Amps For Everyone: Design Reference. Dallas: [s.n.], 2002. 463 p.

MURATA MANUFACTURING CO., LTD. NTC Thermistors. [S.I.], p. 39. 2013.

NARDI, C. Dimensionamento de Filtros passivos para conversores estáticos de energia. Pato Branco: [s.n.], 2016. 162 p.

NATIONAL SEMICONDUCTOR. LM35: Precision Centigrade Temperature Sensors. [S.I.], p. 13. 2000.

PAARMANN, L. D. Design and Analysis of Analog Filter: A Signal Processing Perspective. [S.I.]: Kluwer Academic Publishers, 2003. 440 p.

PAITER, L. Sensor para análise das características físico-químicas de óleo de soja por meio da constante dielétrica. [S.I.]: [s.n.], 2015. 88 p.

PERTENCE JUNIOR, A. Amplificadores Operacionais e Flltros Ativos. Porto Alegre: Bookman, 2015.

THEDE, L. Practical Analog and Digital Filter Design. [S.I.]: Artech House, INC, 2004. 267 p.

WINDER, S. Analog and Digital Filter Design. 2ª ed. [S.I.]: NEWNES, 2002. 450 p. 